\title{
Coupled mesoscopic superconductors: Ginzburg-Landau theory
}

\author{
B. J. Baelus, S. V. Yampolskii, ${ }^{*}$ and F. M. Peeters ${ }^{\dagger}$ \\ Departement Natuurkunde, Universiteit Antwerpen (UIA), Universiteitsplein 1, B-2610 Antwerpen, Belgium
}

(Received 7 December 2001; revised manuscript received 11 March 2002; published 24 July 2002)

\begin{abstract}
The magnetic coupling between two concentric mesoscopic superconductors with nonzero thickness is studied using the nonlinear Ginzburg-Landau theory. We calculated the free energy, the expelled field, the total field profile, the Cooper-pair density, and the current density distribution. By putting a smaller superconducting disk or ring in the center of a larger ring, the properties change drastically. Extra ground-state transitions are found, where the total vorticity stays the same, but the vorticity of the inner superconductor changes by 1 . Due to the magnetic coupling, the current in the external ring exhibits extra jumps at the transition fields where the vorticity of the inner superconductor changes. In this case, for certain temperatures, re-entrant behavior and switching on and off of the superconducting behavior of the rings are found as a function of the magnetic field. A $H-T$ phase diagram is obtained for the situation where the inner ring has a higher critical temperature than the outer ring. An analytic expression for the magnetic coupling is obtained for thin rings and extreme type-II superconductors.
\end{abstract}

DOI: 10.1103/PhysRevB.66.024517

PACS number(s): 74.20.De, 74.25.Ha, 74.25.Dw

\section{INTRODUCTION}

Single mesoscopic superconducting rings ${ }^{1-11}$ and disks $^{12-25}$ have attracted a lot of attention during the last years. The superconducting properties of these single samples have been studied experimentally and are rather well described and understood theoretically.

Many of these studies were limited to disks ${ }^{12,13,20-23}$ and rings ${ }^{7,9}$ of zero thickness. In such a case the magnetic field induced by the supercurrents are neglected and one assumes that the total field equals the external field, which is taken uniform. In the present paper disks and rings with finite thickness are considered. In this case the superconductor tries to expel the field. Supercurrents are induced creating a local magnetic field opposite to the external one. This leads to a decrease of magnetic field inside the superconductor and an increase near the sample edges. For sufficiently large disks and magnetic fields, the magnetic field penetrates the superconductor and vortices are created, which corresponds to regions of local-field compression. In small disks, only circular symmetric vortex states nucleate in the center, which are called giant vortex states. Encircling the vortex on a closed loop, the phase change of the order parameter equals $L$ times $2 \pi$, where $L$ is the angular momentum or vorticity. In larger disks, seperate vortices can nucleate creating a multivortex state and the vorticity $L$ is just the number of vortices. In the case of rings, the magnetic field can be expelled to the inside or to the outside of the ring or partially to both sides.

In the present paper we want to understand what will happen if two mesoscopic superconductors are put close to each other but are electrically isolated. When a uniform magnetic field is applied, it will be locally altered by each of the superconductors. In some regions of space the field will be expelled from the superconducting disk or ring, while in other regions it will be compressed into vortices penetrating the sample or compressed into the inside of a superconducting ring. This results in a strongly nonuniform total field, which is the superposition of the applied field and the field created by the supercurrents. Another superconductor will interact with this nonuniform field. In this way the superconductors are coupled by the magnetic field and they interact with each other. This coupling will influence the properties of the two superconductors. It is this coupling that will be studied in the present paper.

The magnetic coupling between normal mesoscopic rings is well known. Wang et al. ${ }^{26}$ studied theoretically the persistent currents of $N$ normal rings placed periodically on the same plane. Because of the mutual inductance between two rings, the electric current in one ring produces an induced flux in the other ring, creating an extra current in this ring. They found that the mutual inductance between normal rings enhances the persistent current as was the case in the experiments described in Ref. 27.

Correlations in arrays of magnetically coupled superconducting aluminum rings were investigated experimentally by Davidović et al. ${ }^{28}$ They used ultrasensitive susceptibility techniques and scanning Hall probe microscopy to study arrays of electrically isolated superconducting rings of micron size. When the external flux is close to $\Phi_{0} / 2$, the magnetic moments produced by the supercurrents in such rings are analogous to Ising spins. Magnetic moments parallel to the applied magnetic field can be called "spin up," while those in the opposite direction "spin down." Via their dipolar magnetic fields, neighboring rings can interact antiferromagnetically and the different rings influence each other.

Recently, Morelle et al. ${ }^{29}$ studied experimentally the magnetic interaction between two superconducting mesoscopic aluminum rings, close to the superconducting/normal phase transition. In their sample, a smaller ring was placed in the center of the larger ring. Using resistivity measurements the phase boundary was obtained for the two-ring structure as well as for the reference single ring. In both systems, LittleParks oscillations were observed in the $H-T$ phase diagram. The modification of the $T_{c}(H)$ oscillations of the outer ring is seen in the Fourier spectrum of the $T_{c}(H)$ line due to the 

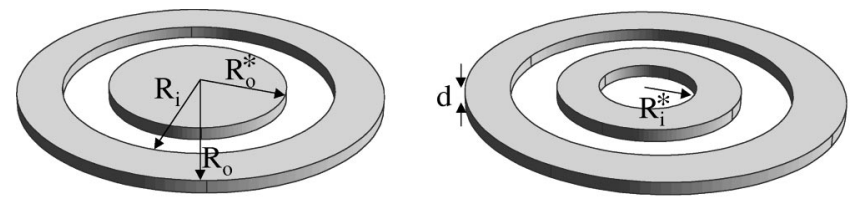

FIG. 1. Schematical outline of the considered configurations; the ring-disk configuration (left) and the ring-ring configuration (right).

coupling between the outer and the inner ring. They suggested that an inner ring made from a different superconductor with a higher critical temperature would increase the magnetic coupling between the two rings.

In the present paper we present a theoretical investigation of the properties of two coupled mesoscopic superconductors. Our main attention will go to the interaction between the two superconductors. How they influence each other? How do the superconducting properties of a single ring change when another superconductor is placed in its center? Therefore we consider two different configurations: (i) a ring-disk configuration where a small disk is placed in the center of a larger ring, and (ii) a ring-ring configuration where a small ring is placed in the center of the larger ring as in the experiment of Ref. 29. We will also give an example of a ring-ring system where the inner ring is made from a different superconductor with a higher critical temperature. Our theoretical analysis is based on a full self-consistent numerical solution of the coupled nonlinear Ginzburg-Landau equations. Since we consider sufficiently narrow rings and small disks, only axial symmetric giant vortex states will nucleate. ${ }^{8}$ Therefore the equations can be solved for a fixed value of the vorticity. The magnetic-field profile outside and inside the superconductor is obtained self-consistently and therefore the full demagnetization effect is included in our approach.

The paper is organized as follows: In Sec. II we describe the theoretical formalism. Our results for the ring-disk configuration are discussed in Sec. III. In Sec. IV the ring-ring configuration is studied. In Sec. V we calculate the $H-T$ phase diagram for the two ring system, where the inner ring is made of a different material with a higher critical temperature. In Sec. VI we analytically calculate the energy of two coupled thin rings made of a different material for high values of $\kappa$. Finally, in Sec. VII our results are summarized.

\section{THEORETICAL FORMALISM}

We consider a superconducting ring with inner radius $R_{i}$, outer radius $R_{o}$, and thickness $d$ immersed in an insulating medium (for example, vacuum). In the center of this ring a superconducting disk with radius $R_{o}^{*}$ or another superconducting ring with inner radius $R_{i}^{*}$ and outer radius $R_{o}^{*}$ is placed with the same thickness (see Fig. 1). The whole sample is placed in a perpendicular uniform magnetic field $\vec{H}=\left(0,0, H_{0}\right)$. To solve this problem, we expand our previous approach for thin superconducting disks ${ }^{17}$ to a system of two axial symmetric superconductors each made of a different material. In the present paper we solve the system of two coupled nonlinear Ginzburg-Landau equations which determine the distribution of both the superconducting order pa- rameter $\Psi(\vec{r})$ and the vector potential $\vec{A}(\vec{r})$ inside and outside the superconductor,

$$
\begin{gathered}
\frac{1}{2 m}\left(-i \hbar \vec{\nabla}-\frac{2 e \vec{A}}{c}\right)^{2} \Psi=-\alpha_{i, o} \Psi-\beta_{i, o} \Psi|\Psi|^{2}, \\
\vec{\nabla} \times \vec{\nabla} \times \vec{A}=\frac{4 \pi_{\vec{j}}}{c},
\end{gathered}
$$

where for the inner superconductor the parameters are $\alpha_{i, o}$ $=\alpha_{i}, \beta_{i, o}=\beta_{i}$, and for the outer ring $\alpha_{i, o}=\alpha_{o}, \beta_{i, o}=\beta_{o}$. The density of superconducting current $\vec{j}$ is given by

$$
\vec{j}=\frac{e \hbar}{i m}\left(\Psi^{*} \vec{\nabla} \Psi-\Psi \vec{\nabla} \Psi^{*}\right)-\frac{4 e^{2}}{m c}|\Psi|^{2} \vec{A},
$$

and $m$ is the mass of the Cooper pair. Since we only consider circular symmetric rings and disks, we use cylindrical coordinates. Any position in space will be expressed by $\vec{r}$ $=(\rho, \phi, z)$, where $\rho$ is the radial distance from the center, $\phi$ is the azimuthal angle, and $z$ is the perpendicular direction. The sample lies between $z=-d / 2$ and $z=d / 2$.

Equations (1a) - (2) have to be supplemented by boundary conditions for $\Psi(\vec{r})$ and $\vec{A}(\vec{r})$. The boundary condition for the superconducting condensate at the superconductor/ insulator surfaces is given by

$$
\left.\vec{n} \cdot\left(-i \hbar \vec{\nabla}-\frac{2 e \vec{A}}{c}\right)\right|_{\rho=R_{1}^{*}, R_{2}^{*}, R_{1}, R_{2}} \Psi=0,
$$

where $\vec{n}$ is the unit vector in the radial direction. This condition expresses that the superconducting current in the radial direction vanishes at the sample surface. The boundary for the vector potential has to be taken far away from the disk,

$$
\left.\vec{A}\right|_{\vec{r} \rightarrow \infty}=\frac{1}{2} \vec{e}_{\phi} H_{0} \rho,
$$

where the field equals the applied magnetic field $\vec{H}$ $=\left(0,0, H_{0}\right)$.

To rewrite Eqs. (1a)-(2) in dimensionless variables, we express all distances in units of the coherence length of the outer superconductor $\xi_{o}=\hbar / \sqrt{-2 m \alpha_{o}}$, the order parameter in $\Psi_{0, o}=\sqrt{-\alpha_{o} / \beta_{o}}$, the vector potential in $c \hbar / 2 e \xi_{o}$, and the magnetic field in $H_{c 2, o}=c \hbar / 2 e \xi_{o}^{2}=\kappa_{o} \sqrt{2} H_{c, o}$, where $H_{c, o}$ $=\sqrt{4 \pi \alpha_{o}^{2} / \beta_{o}}$ is the thermodynamical critical field of the outer superconductor and $\kappa_{o}=\lambda_{o} / \xi_{o}$ is the Ginzburg-Landau parameter for this superconductor. The penetration depth of the outer ring is given by $\lambda_{o}=\sqrt{m / \pi}(c / 4 e) / \Psi_{0, o}$. Using these dimensionless variables and the London gauge, $\operatorname{div} \vec{A}$ $=0$, we can rewrite Eqs. (1a)-(2) in the following form:

$$
(-i \vec{\nabla}-\vec{A})^{2} \Psi=\Psi\left(\frac{\xi_{o}^{2}}{\xi_{i, o}^{2}}-\frac{\kappa_{i, o}^{2}}{\kappa_{o}^{2}}|\Psi|^{2}\right),
$$




$$
-\kappa_{o}^{2} \nabla^{2} \vec{A}=\frac{1}{2 i}(\Psi * \vec{\nabla} \Psi-\Psi \vec{\nabla} \Psi *)-|\Psi|^{2} \vec{A},
$$

where $\xi_{i, o}=\xi_{i}, \kappa_{i, o}=\kappa_{i}$ in the inner superconductor, and $\xi_{i, o}=\xi_{o}, \kappa_{i, o}=\kappa_{o}$ in the outer ring. The first boundary condition becomes

$$
\left.\vec{n} \cdot(-i \vec{\nabla}-\vec{A})\right|_{\rho=R_{1}^{*}, R_{2}^{*}, R_{1}, R_{2}} \Psi=0 .
$$

Provided that Eqs. (1a)-(2) are fulfilled, the difference between the Gibbs free energy of the superconducting state and the normal state is determined by the expression

$$
F=\frac{1}{V} \int\left[2\left(\vec{A}-\vec{A}_{0}\right) \cdot \vec{j}-\frac{\kappa_{i, o}^{2}}{\kappa_{o}^{2}}|\Psi|^{4}\right] d \vec{r},
$$

where the integral is over the total volume $V$ of the superconducting samples and $\vec{A}_{0}=\frac{1}{2} \vec{e}_{\phi} H_{0} \rho$ is the external vector potential in the absence of a superconductor. The free energy is measured in $H_{c, o}^{2} V / 8 \pi$. The dimensionless supercurrent $\vec{j}$ is given by $\vec{j}=\left(\Psi * \vec{\nabla} \Psi-\Psi \vec{\nabla} \Psi^{*}\right) / 2 i-|\Psi|^{2} \vec{A}$.

We restrict ourselves to samples with thickness $d<\xi$, which implies that we are allowed to assume that the order parameter does not vary in the $z$ direction. ${ }^{15,17}$ On the other hand, for the vector potential the variation in the $z$ direction is retained and for $d>\lambda$ this is very important due to the Meissner effect. ${ }^{15,17}$

We consider sufficiently narrow rings and small disks, and therefore only axial symmetric giant vortex states will nucleate. ${ }^{8,17}$ Consequently, the equations can be solved for a fixed value of the angular momentum $L_{\text {out }}$ in the outer ring and $L_{i n}$ in the inner superconductor that leads to the order parameter

$$
\Psi(\rho, \phi)=f(\rho) e^{i L_{\text {in,out }} \phi},
$$

where $L_{\text {in,out }}=L_{\text {out }}$ in the outer ring and $L_{\text {in out }}=L_{\text {in }}$ in the inner superconductor. Consequently, both the vector potential and the superconducting current density are directed along the azimuthal direction $\vec{e}_{\phi}$. For fixed angular momenta $L_{\text {out }}$ and $L_{i n}$, Eqs. (5a) and (5b) can be reduced to the following form:

$$
\begin{gathered}
\frac{-1}{\rho} \frac{\partial}{\partial \rho} \rho \frac{\partial f}{\partial \rho}+\left\langle\left(\frac{L_{\text {in out }}}{\rho}-A\right)^{2}\right\rangle f=f\left(\frac{\xi_{o}^{2}}{\xi_{i, o}^{2}}-\frac{\kappa_{i, o}^{2}}{\kappa_{o}^{2}} f^{2}\right), \\
-\kappa_{o}^{2}\left(\frac{\partial}{\partial \rho} \frac{1}{\rho} \frac{\partial \rho A}{\partial \rho}+\frac{\partial^{2} A}{\partial z^{2}}\right)=\left(\frac{L_{\text {in }, \text { out }}}{\rho}-A\right) f^{2} \theta\left(\frac{2|z|}{d}\right),
\end{gathered}
$$

where $\theta(x)=1$ for $x<1$ and $\theta(x)=0$ for $x>1, \vec{A}=A \vec{e}_{\phi}$, and \langle\rangle indicates averaging over the disk thickness $\langle f(\vec{r})\rangle$ $=(1 / d) \int_{-d / 2}^{+d / 2} f(z, \rho) d z$.

Because the superconducting condensates of the inner and outer superconductors are disconnected from each other they cannot influence each other directly. The coupling is entirely due to the magnetic field, or equivalently the vector potential, as is expressed by the second term in Eq. (8a). The total magnetic field is a sum of the applied field and the field created by the superconducting currents of the inner ring and the outer superconductor which is described by Eq. (8b) where $f(\rho)=f_{\text {in }}(\rho)+f_{\text {out }}(\rho)$ and $f_{\text {in }}(\rho)\left[f_{\text {out }}(\rho)\right]$ is only different from zero in the interval $R_{i}^{*}<\rho<R_{o}^{*}\left[R_{i}<\rho\right.$ $\left.<R_{o}\right]$.

The magnetic field created by the supercurrent has a $H$ $\sim 1 / r^{3}$ dependence for large $r$. Therefore we can restrict our calculations to a region with radial size $R_{s}$ which we took typically five times the sample size and longitudinal size $d_{s}$ typically ten times the sample thickness. The boundary conditions for the outer parameter can be written as

$$
\left.\frac{\partial f}{\partial \rho}\right|_{\rho=R_{1}^{*}, R_{2}^{*}, R_{1}, R_{2}}=0,
$$

for the ring-ring configuration, and

$$
\left.\frac{\partial f}{\partial \rho}\right|_{\rho=R_{2}^{*}, R_{1}, R_{2}}=0,\left.\quad \rho \frac{\partial f}{\partial \rho}\right|_{\rho=0}=0
$$

for the ring-disk configuration. The last condition of Eq. (10) follows from the rotational symmetry of the system. The current density has to vanish in the center of the disk. The condition (4) for the vector potential taken at infinity can be transferred to the boundaries of our simulation region,

$$
A\left(z, \rho=R_{s}\right)=\frac{1}{2} H_{0} R_{s}, A\left(|z|=d_{s}, \rho\right)=\frac{1}{2} H_{0} \rho .
$$

Following the approach of Ref. 17 we apply a finite difference representation on the space grid $\rho_{n}, z_{m}$ to solve Eqs. (8a) and (8b). Since the size of our simulation region exceeds by far those of the sample, we apply nonuniform space grids to diminish the computer time. The space grid is taken uniform inside the sample, and we increase the grid spacing exponentially with distance outside the sample. This allows us to use the same number of grid points, typically 128 , inside and outside the sample. To obtain steady-state solutions for a system of two superconductors, the following iteration procedure was used:

$$
\begin{aligned}
\eta_{f} f_{n}^{k} & -\frac{2}{\rho_{n+1 / 2}^{2}-\rho_{n-1 / 2}^{2}} \\
& \times\left(\rho_{n+1 / 2} \frac{f_{n+1}^{k}-f_{n}^{k}}{\rho_{n+1}-\rho_{n}}-\rho_{n-1 / 2} \frac{f_{n}^{k}-f_{n-1}^{k}}{\rho_{n}-\rho_{n-1}}\right) \\
& +\left\langle\left(\frac{L_{\text {in,out }}}{\rho}-A\right)^{2}\right) f_{n}^{k}-\frac{\xi_{o}^{2}}{\xi_{i, o}^{2}} f_{n}^{k}+3 \frac{\kappa_{i, o}^{2}}{\kappa_{o}^{2}}\left(f_{n}^{k-1}\right)^{2} f_{n}^{k} \\
& =\eta_{f} f_{n}^{k-1}+2 \frac{\kappa_{i, o}^{2}}{\kappa_{o}^{2}}\left(f_{n}^{k-1}\right)^{3},
\end{aligned}
$$




$$
\begin{aligned}
\eta_{a} A_{m, n}^{k}-\frac{2 \kappa_{o}^{2}}{\rho_{n+1 / 2}-\rho_{n-1 / 2}} \\
\quad \times\left(\frac{\rho_{n+1} A_{m, n+1}^{k}-\rho_{n} A_{m, n}^{k}}{\rho_{n+1}^{2}-\rho_{n}^{2}}-\frac{\rho_{n} A_{m, n}^{k}-\rho_{n-1} A_{m, n-1}^{k}}{\rho_{n}^{2}-\rho_{n-1}^{2}}\right) \\
-\frac{2 \kappa_{o}^{2}}{z_{m+1 / 2}-z_{m-1 / 2}}\left(\frac{A_{m+1, n}^{k}-A_{m, n}^{k}}{z_{m+1}-z_{m}}-\frac{A_{m, n}^{k}-A_{m-1, n}^{k}}{z_{m}-z_{m-1}}\right) \\
-\left(\frac{L_{\text {in,out }}}{\rho_{n}}-A_{m, n}^{k}\right)\left(f_{n}^{k}\right)^{2}=\eta_{a} A_{m, n}^{k-1},
\end{aligned}
$$

where $A_{m, n}=A\left(z_{m}, \rho_{n}\right), f_{n}=f\left(\rho_{n}\right), \rho_{n+1 / 2}=\left(\rho_{n+1}+\rho_{n}\right) / 2$, and $z_{m+1 / 2}=\left(z_{m+1}+z_{m}\right) / 2$. The upper index $k$ denotes the iteration step. To speed up the convergency we introduced the iteration parameters $\eta_{f}$ and $\eta_{a}$, and we expanded the nonlinear term $\left(f_{n}^{k}\right)^{3}=\left(f_{n}^{k-1}\right)^{3}+3\left(f_{n}^{k-1}\right)^{2}\left(f_{n}^{k}-f_{n}^{k-1}\right)$.

\section{RING-DISK CONFIGURATION}

First, we consider a superconducting ring with a superconducting disk in the center. We investigate the influence of the disk on the properties of the ring. As an example, we take a ring with inner radius $R_{i}=1.5 \xi$ and outer radius $R_{o}$ $=2.0 \xi$ and a disk in the center with radius $R_{o}^{*}=1.0 \xi$. Both superconductors have the same thickness, $d=0.15 \xi$, and the Ginzburg-Landau parameter was taken to be $\kappa=0.28$, which is a typical value for mesoscopic $\mathrm{Al}$ superconductors. ${ }^{14,15}$

Figure 2(a) shows the free energy for the considered system as a function of the applied magnetic field. First we consider the uncoupled system and calculated the free energy for the disk in the center (thick dashed curve) and the free energy for the different giant vortex states in the outer ring (thick dotted curve). The results for single rings and disks were exhaustively described in previous papers. ${ }^{17,8}$ Notice that, in this paper, the free energy is expressed in units of $F_{0}=H_{c}^{2} V / 8 \pi$, where $V$ is the sum of the disk and the ring volume. This is the reason why the free energy of the disk and the ring are not equal to $-F_{0}$ at zero magnetic field as it was in Refs. 17 and 8. The size of the disk is such that only the Meissner state, i.e., the $L_{i n}=0$ state, can nucleate. At applied magnetic fields $H_{0} / H_{c 2} \geq 2.9$ the disk is in the normal state, which results in $F=0$. In the single ring, on the other hand, different giant vortex states with vorticity $L_{\text {out }}$ $=0$ up to $L_{\text {out }}=10$ can nucleate before the ring becomes normal at $H_{0} / H_{c 2} \approx 6.8$. Next, we introduced the magnetic coupling between the disk and the ring and the results are given by the solid curves in Fig. 2(a). The different axial symmetric states are determined by the vorticity of the disk $L_{\text {in }}$ and the total vorticity $L_{\text {out }}$, which is equal to the vorticity of the ring. Therefore we characterize the states by $\left(L_{\text {out }}, L_{\text {in }}\right)$. For the considered configuration, we find states with $L_{\text {in }}=0$ and $L_{\text {out }}=0$ up to $L_{\text {out }}=5$. We also find states with $L_{\text {in }}=1$ and $L_{\text {out }}=0$ up to $L_{\text {out }}=10$, which equal the giant vortex states of the single ring, because the disk is now in the normal state. Notice further that we could also write

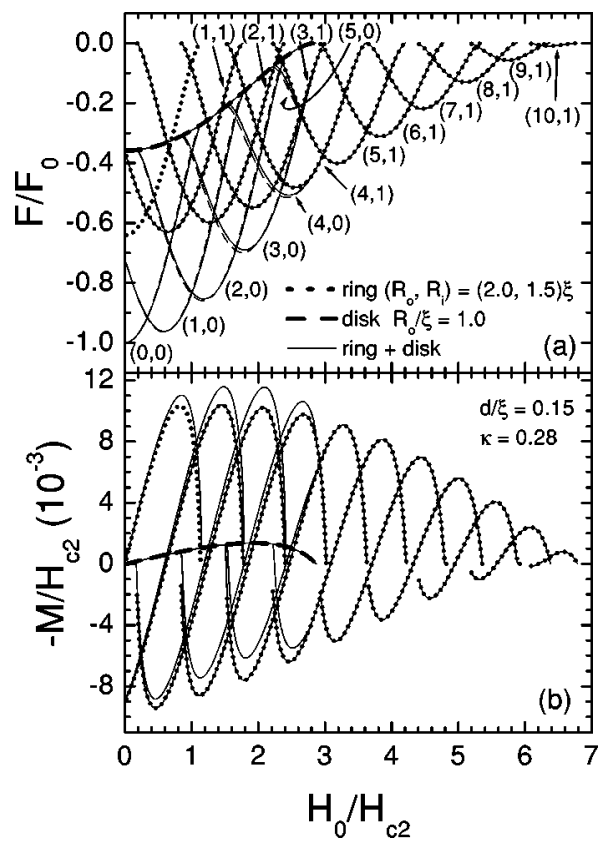

FIG. 2. (a) The free energy and (b) the expelled field as a function of the applied field for a ring with inner radius $R_{i}=1.5 \xi$ and outer radius $R_{o}=2.0 \xi$ (thick dotted curves) and a disk in the center with radius $R_{o}^{*}=1.0 \xi$ (thick dashed curves) and for the coupled ring-dot configuration (thin solid curves). All superconductors have the same thickness, $d=0.15 \xi$, and $\kappa=0.28$. The thin dashed curves give the sum of the free energies of the single disk and the single ring.

$(6,0)$ instead of $(6,1)$ because for the applied magnetic fields where the $L_{\text {out }}=6$ state in the ring exists the disk is normal even for $L_{i n}=0$. We have chosen to write $L_{i n}=1$ because this expresses more clearly that there is flux going through the disk. If both the disk and the ring are superconducting, the free energy of the total sample is different from the sum of the free energies of the single disk and the single ring.

To investigate these new states in more detail we consider as an example the $(2,0)$ state. Figures $3(a)-(c)$ show the magnetic-field distribution, the current density, and the Cooper-pair density, respectively, as a function of the radial position for five different applied magnetic fields, i.e., $H_{0} / H_{c 2}=0.1,0.5,1.5,2.0$, and 2.5. Near $H_{0} / H_{c 2}=0$ the $(2,0)$ state equals the $L_{i n}=0$ state of the disk and the ring is in the normal state. The reason is that the applied field is so low that a lot of magnetic flux has to be attracted to create a state with $L_{\text {out }}=2$ in the outer ring. Therefore a very high superconducting current has to flow through the outer ring which leads to the destruction of superconductivity in this ring. The solid curves in Figs. 3(a)-(c) show that the Cooper-pair density and the current density are indeed zero in the ring. The magnetic-field distribution shows the flux expulsion from the disk. Inside the disk the field decreases and at the edge there is a peak which illustrates a higher concentration of field because of the demagnetization effects. With increasing external field less flux has to be attracted and the current in the outer ring decreases. At $H_{0} / H_{c 2} \approx 0.17$ superconductivity is restored in the external ring [see the dotted curves in Fig. 3(c)]. The dotted curves in Fig. 3(b) 


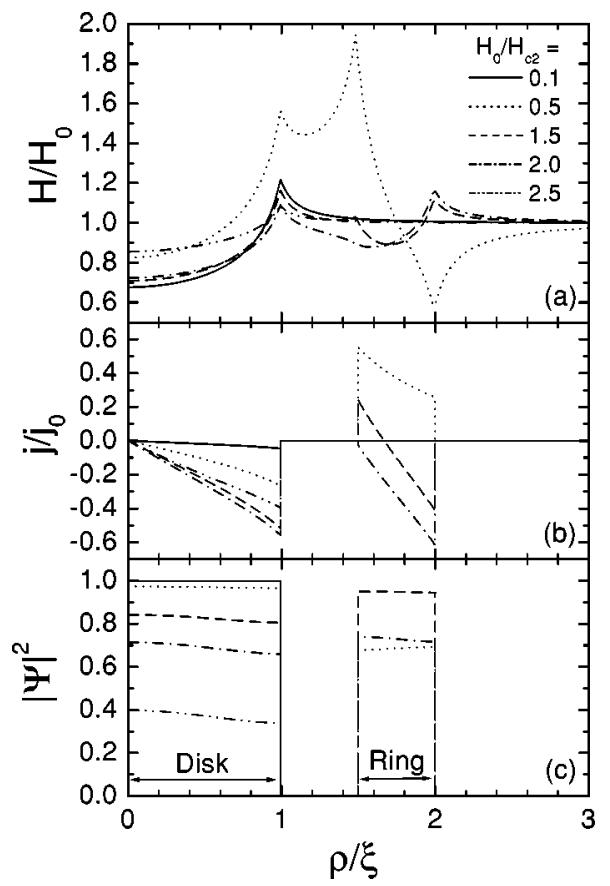

FIG. 3. (a) The magnetic-field distribution, (b) the current density, and (c) the Cooper-pair density as a function of the radial position for the $\left(L_{\text {out }}, L_{i n}\right)=(2,0)$ state of the ring-dot configuration of Fig. 2 at $H_{0} / H_{c 2}=0.1,0.5,1.5,2.0$, and 2.5.

show that the current in the outer ring flows in the opposite direction than the current in the disk. The superconducting currents in the disk expel the flux, while the currents in the ring are attracting flux, which is compressed in the region between the disk and the ring [see the dotted curve in Fig. $3(\mathrm{a})]$. The free energy becomes now more negative as compared to the free energy of the single disk [see Fig. 2(a)]. Increasing the magnetic field further leads to less attraction of flux and hence to a higher Cooper-pair density in the ring and a more negative free energy. When the external flux becomes comparable with the flux needed for the $L_{\text {out }}=2$ state, the outer part of the ring expels the flux to the outside, while the inner part of the ring still expels flux to the hole region. Therefore the superconducting current changes sign in the ring region [see the dashed curve in Fig. 3(b)]. Since the flux is expelled in both directions, the dashed curve in Fig. 3(a) shows a positive peak at both ring boundaries. Further increasing the external field leads to external fluxes larger than the flux needed for the $L_{\text {out }}=2$ state and hence the ring has to expel flux in order to keep vorticity $L_{\text {out }}=2$. As a consequence, the current in the ring has to flow in the same direction as the current in the disk, which is shown by the dash-dotted curve in Fig. 3(b). Because of the expulsion, the field between the two superconductors is lower than the external field [see the dash-dotted curve in Fig. 3 (a)]. If we further increase the magnetic field, the superconducting currents in the outer ring have to increase in order to expel more flux and consequently the Cooper-pair density decreases in the outer ring. At $H_{0} / H_{c 2} \approx 2.4$ the supercurrent becomes too high and the ring becomes normal again [see the dash-dotdotted curves in Figs. 3(a)-(c)]. At this field, the free energy equals the free energy of the single disk.
The above discussion shows clearly the interplay between the superconducting state of the disk and the ring. Next, we investigate the interaction between the disk and the ring. Therefore we added the sum of the free energies of the single disk and the single ring [thin dashed curves in Fig. 2(a)] and compare this result with the result of the ring-disk configuration [solid curves in Fig. 2(a)]. Notice that there is a small difference between the two set of curves, which is due to the coupling between the two superconductors. Below we will show that this difference becomes more pronounced for thicker samples.

Now, we will determine the attraction or expulsion of the magnetic field by the coupled superconducting system. Figure 2(b) shows the magnetic field expelled from the sample, $-M$, as a function of the applied magnetic field:

$$
M=\frac{\langle H\rangle-H_{0}}{4 \pi},
$$

where $\langle H\rangle$ is the magnetic field averaged over the area $\rho$ $<R_{o}$, i.e., the outer radius of the ring and $H_{0}$ is the applied field. The thick dashed and thick dotted curves are the results for the single disk and the single ring and the solid curves for the total ring-disk system. By putting a disk in the center of the ring, more field is expelled and less field is attracted. Of course, for $H_{0} / H_{c 2} \gtrsim 2.9$ the disk is in the normal state and we recover the result for the single ring case.

\section{RING-RING CONFIGURATION}

In this section we replace the disk in the center by a second ring and the influence of this inner ring on the outer ring will be investigated. As an example for this ring-ring configuration, we consider a superconducting ring with inner radius $R_{i}=1.5 \xi$ and outer radius $R_{o}=2.0 \xi$ and a second ring in the center with inner radius $R_{i}^{*}=0.6 \xi$ and outer radius $R_{o}^{*}=1.1 \xi$. Both rings have the same thickness, $d=0.15 \xi$ and the same Ginzburg-Landau parameter $\kappa=0.28$.

Figure 4 shows the free energy as a function of the applied magnetic field for the small single ring by thick dashed curves, for the larger single ring by thick dotted curves, and for the coupled ring-ring situation by the thin solid curves, where the interaction between the two rings is taken into account. In the inner ring superconducting states can nucleate with vorticity $L_{i n}=0,1$, and 2 and the superconducting/ normal transition field is at $H_{0} / H_{c 2} \approx 6.4$. In the outer ring states with vorticity $L_{\text {out }}=0$ up to $L_{\text {out }}=10$ exist and superconductivity is destroyed at $H_{0} / H_{c 2} \approx 6.75$. The superconducting states nucleating in the double ring system can be characterized again by the indices $\left(L_{\text {out }}, L_{i n}\right)$. For $L_{\text {in }}=0$, superconducting states can nucleate with $L_{\text {out }}=0$ up to 4 , for $L_{\text {in }}=1$ with $L_{\text {out }}=1$ up to $L_{\text {out }}=8$, and for $L_{\text {in }}=2$ with $L_{\text {out }}=5$ up to 10 . For $L_{\text {in }} \geqslant 3$ the states equal the states of the single outer ring because the inner ring will be normal.

The indices in the figure correspond to the ground state of the coupled ring system. For the numerical example studied in the previous section the number and the position of the ground-state transitions are the same as for the outer ring. In 


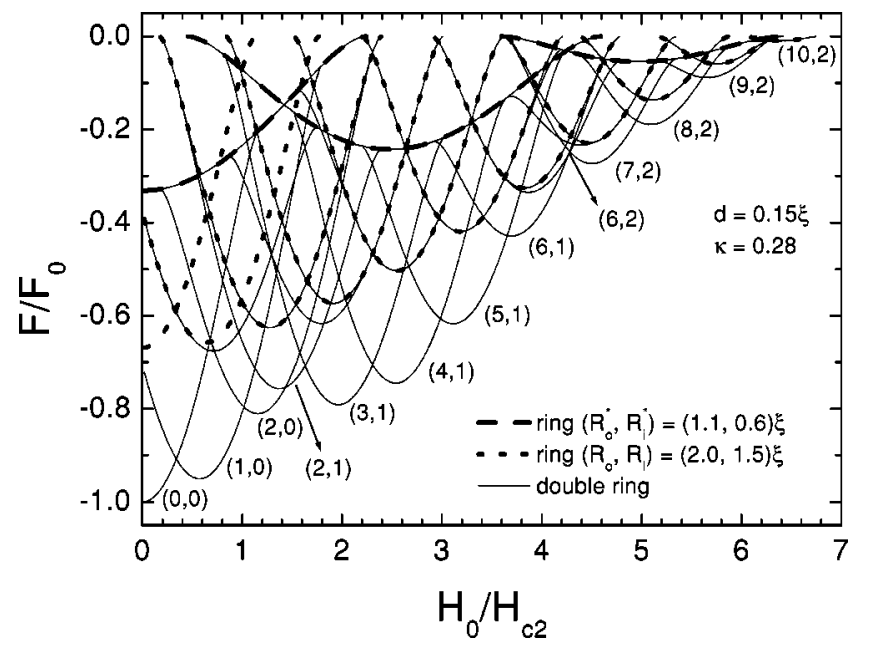

FIG. 4. The free energy as a function of the applied magnetic field for a superconducting ring with radii $R_{i}=1.5 \xi$ and $R_{o}=2.0 \xi$ (dotted curves), a ring with $R_{i}^{*}=0.6 \xi$ and $R_{o}^{*}=1.1 \xi$ (dashed curves) and the double ring configuration (solid curves). Both rings have the same thickness, $d=0.15 \xi$, and $\kappa=0.28$. The indices indicate the ground-state vorticities $\left(L_{\text {out }}, L_{i n}\right)$.

the present two ring system this is no longer the case and the number of transitions in the coupled system are larger than for the single outer ring case. The inner ring induces extra transitions in the coupled system each time when the vorticity of the inner ring changes with one unit. The first extra transition is the transition from $(2,0)$ to $(2,1)$ and the second one is the transition from $(6,1)$ to $(6,2)$. The $(2,1)$ state is the ground state in the magnetic field region $1.43 \leqslant H_{0} / H_{c 2}$ $\lesssim 1.63$ and the $(6,2)$ state in the region $4.27 \lesssim H_{0} / H_{c 2}$ $\$ 4.28$. Hence, by putting a ring in the center of the larger ring, the ground state shows extra transitions. This result corresponds to the experimental result of Morelle et al., ${ }^{29}$ who saw modifications of the $T_{c}(H)$ oscillations of the outer ring in the Fourier spectrum of the $T_{c}(H)$ line due to the coupling between the outer and the inner ring.

Next, we focus further on the interaction between the inner ring and the outer ring. Therefore we plot in Fig. 5 the ground-state free energy of the coupled rings (solid curves) and the sum of the free energies of the two single rings (dashed curves) for the previous configuration (upper curves which are shifted by +0.1 ), i.e., $d=0.15 \xi$, and for a thicker sample with $d=1.0 \xi$ (lower curves). For $d=0.15 \xi$ the difference is most pronounced for the $(2,0)$, the $(5,1)$, and the $(6,1)$ state. Both the value of the free energy and the transition magnetic fields are influenced by the interaction between the two rings. The left inset shows the $(6,1) \rightarrow(6,2)$ $\rightarrow(7,2)$ transition in more detail. Notice that the interaction significantly decreases the magnetic field region where the $(6,2)$ state is the ground state. For $d=1.0 \xi$ the demagnetization effects become more important and therefore the interaction between the two rings gains importance. This results in a larger difference between the dashed and solid curves. The value of the free energy and the transition fields are changing considerably by the fact that both rings are influencing each other. The two lower insets show the $(2,0)$ $\rightarrow(2,1) \rightarrow(3,1)$ and the $(6,1) \rightarrow(7,2)$ transitions in more de-

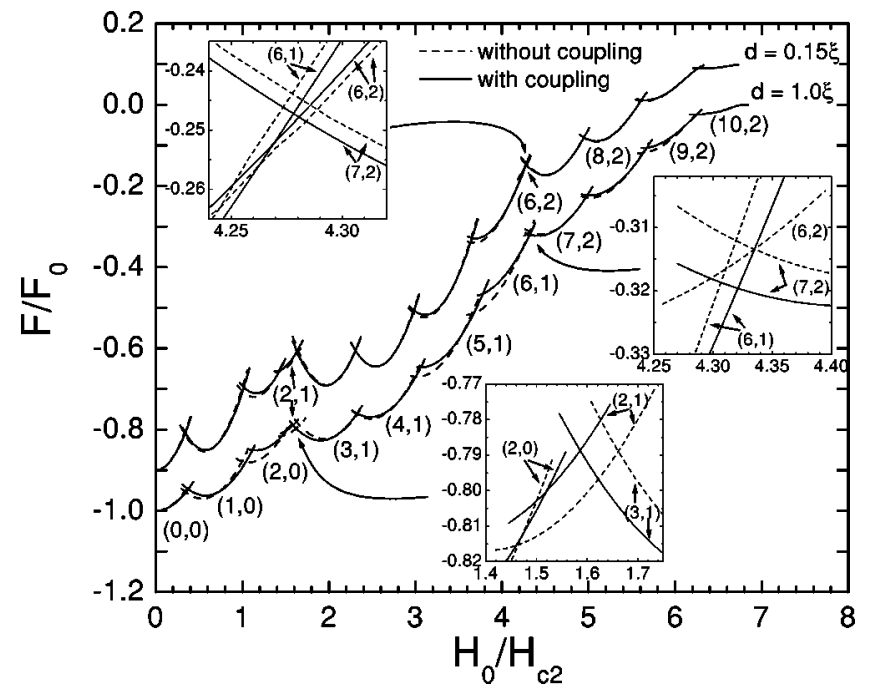

FIG. 5. The ground-state free energy of the double ring configuration of Fig. 4 (solid curves) and the sum of the free energies of the two rings (dashed curves) for $d=0.15 \xi$ (upper curves) and for $d$ $=1.0 \xi$ (lower curves). The upper curve is shifted by +0.1 for clarity. The insets show some of the crossings in more detail.

tail. The magnetic-field region where the ground state is given by the $(2,1)$ state decreases due to the interaction. For the $(6,1) \rightarrow(7,2)$ transition coupling between the two rings leads to the interesting result that the $(6,2)$ state is no longer a ground state.

Figures 6(a) and (b) show the magnetic field expelled from the sample, $-M$, as a function of the applied magnetic field for the single outer ring (dashed curve) and for the

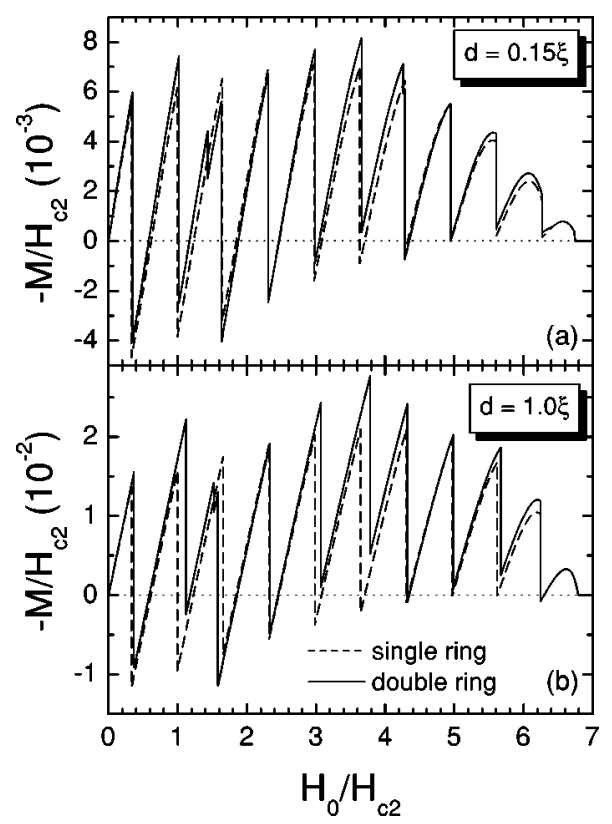

FIG. 6. The magnetic field expelled from the sample, $-M$, as a function of the applied magnetic field for the single outer ring (dashed curve) and for the double ring configuration (solid curve). The sample is the same as in Fig. 4 with thickness $d=0.15 \xi$ (a) and $d=1.0 \xi(\mathrm{b})$. 


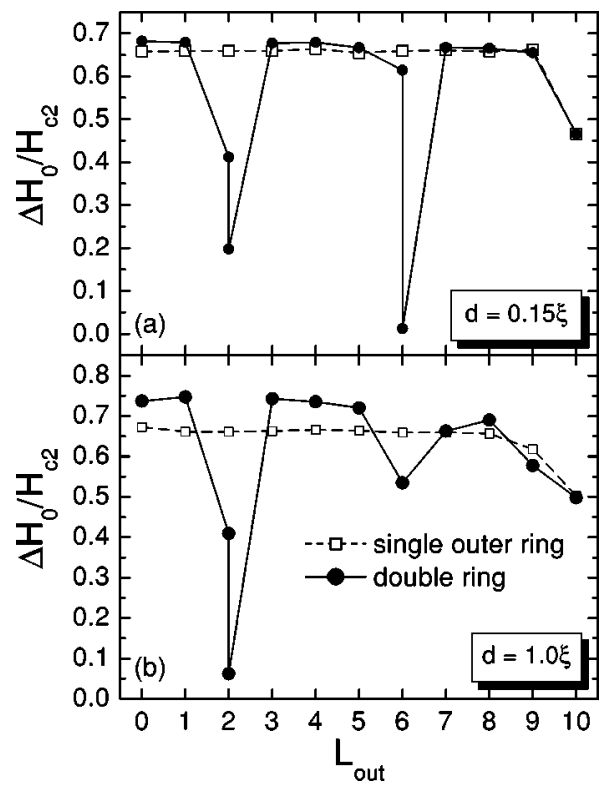

FIG. 7. The magnetic-field range $\Delta H_{0}$ over which the $\left(L_{\text {out }}, L_{\text {in }}\right)$ state is the ground state, as a function of $L_{\text {out }}$ for the single outer ring (the dashed curve and open squares) and for the double ring system (the solid curve and closed circles). The sample is the same as in Fig. 4 with thickness $d=0.15 \xi$ (a) and $d=1.0 \xi$ (b).

double ring configuration (solid curve) for thickness $d$ $=0.15 \xi$ and $d=1.0 \xi$, respectively. For $d=0.15 \xi$, the two extra transitions, resulting from the influence of the inner ring, are clearly visible by the jumps at $H_{0} / H_{c 2}=1.43$ and 4.28. Notice that, depending on the direction of the current, a single ring expels or attracts field at a given applied magnetic field and vorticity. Expulsion (attraction) leads to a lower (higher) magnetic-field density in the center of the ring and to a higher (lower) density near the outside. Therefore, depending on the applied field, the field expulsion or attraction in the coupled ring configuration can either increase if the two currents are in the same direction, or decrease if the directions are opposite. This can be clearly seen from Figs. 6(a) and (b) where the field expulsion or attraction becomes more pronounced by putting the inner ring in the center of the outer ring. For $d=1.0 \xi$ one extra transition results from the influence of the inner ring, i.e., at $H_{0} / H_{c 2}=1.58$. The difference between the transition fields of the single ring and the coupled ring system becomes larger (see also the lower curve in Fig. 5) and the difference in the expulsion becomes more pronounced with increasing the sample thickness, which indicates again that the interaction between the two rings increases with increasing $d$.

In Figs. 7(a) and (b) the magnetic-field range $\Delta H_{0}$ over which the $\left(L_{\text {out }}, L_{\text {in }}\right)$ state is the ground state is plotted as a function of $L_{\text {out }}$ for thickness $d=0.15 \xi$ and $d=1.0 \xi$, respectively. This magnetic-field range corresponds to the distance between two consecutive jumps in the expelled field (see Fig. 6). The results for the single outer ring are given by the open squares and for the double ring system by the closed circles. The curves are guides to the eye. For $d=0.15 \xi$ the

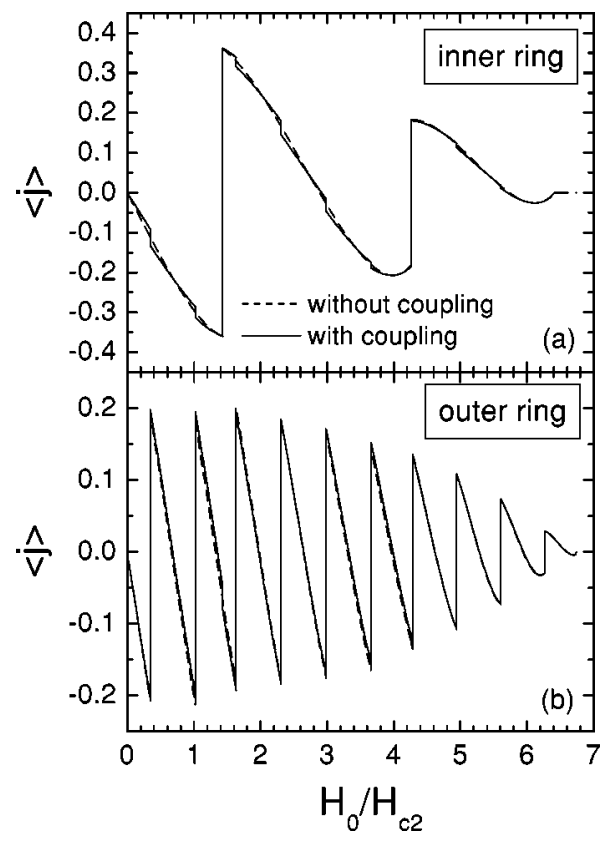

FIG. 8. The averaged current density for the ground state in the inner ring (a) and the outer ring (b) as a function of the applied magnetic field for the same double ring configuration as in Fig. 4. The results for the single rings are given by dashed curves, those for the double ring configuration by solid curves.

extra transitions are clearly visible at $L_{\text {out }}=2$ and 6 and for $d=1.0 \xi$ at $L_{\text {out }}=2$ where for the same $L_{\text {out }}$ two jumps occur due to a transition of the inner ring. Also for the other vorticities $L_{\text {out }}$, there is a difference between $\Delta H_{0}$ for the single ring and the double ring. The reason is that the ground-state transition fields are influenced by the interaction between the two rings. This was also visible in Fig. 5. If the free energy of the double ring was just the sum of the free energies of the two single rings, $\Delta H_{0}$ would be the same for the single outer ring and the double ring, except for $L_{\text {out }}=2$ and 6, where extra transitions occur because $L_{i n}$ changes with one unit. Notice further that the difference between the results for the single outer ring and the double ring enhances with increasing sample thickness.

Next, we investigate the effect of the interaction between the two rings on the superconducting current density in the two rings for $d=0.15 \xi$. Figures 8 (a) and (b) show the averaged current density for the ground state in the inner ring and the outer ring, respectively, as a function of the applied magnetic field. The results for the single ring are given by dashed curves, these for the double ring configuration by solid curves. First, we describe what happens if there is no interaction between the two rings. In this case we can consider them as two single rings. At low fields, the ground state of a single ring is given by the $L=0$ state or Meissner state and the ring expels the field to the outside of the sample. With increasing external field, more flux has to be expelled from the ring which leads to a higher current density. After the first transition the ground state is given by the $L=1$ state and initially flux will be trapped in the ring and the flux going through the ring is larger than the flux of the external field. To compress this extra magnetic field, the superconducting 
current in the ring has to flow in the opposite direction. At the transition, the current shows a jump from a negative to a positive value, i.e., from expulsion to compression. With increasing external field, less flux has to be compressed to achieve vorticity 1 and the current density in the outer ring decreases. Further increasing the field, the external flux becomes larger than the flux needed for $L=1$ and flux has to be expelled. Therefore the current in the ring changes sign. Without interaction between the two rings, the current density in one ring exhibits only jumps when the vorticity of the ground state of this ring changes [see dashed curves in Figs. $8(a)$ and (b)].

In the coupled two rings situation $\langle j\rangle$ shows small jumps on top of the previously described expulsion $\rightarrow$ compression jumps. At low fields, the ground state is given by the $(0,0)$ state or Meissner state. Both rings expel the field to the outside of the sample, which means that the current flows in the same direction in each ring. Since some flux is already expelled by the outer ring, the inner ring has to expel less and therefore the current is less negative. After the first transition the ground state changes into the $(1,0)$ state. Now, the outer ring compresses the field to achieve vorticity 1 , and, as a consequence, the field in the hole of the outer ring is larger than the external field. This means that the inner ring has to expel more field and the current density jumps to a value more negative than its value without interaction. The other transitions can be explained analogously. From Figs. 8(a) and (b) it is clear that the two rings are influencing each other and that the interaction between the two rings results in extra jumps in the current density in one ring when the vorticity of the other ring changes. These jumps are smaller than the jumps when the vorticity of the considered ring increases, but they are not negligible.

Up to now, we considered rather small samples. For the single ring it is known that by increasing the sample size (i) more $L$ states are possible and (ii) the magnetic-field range over which the state with vorticity $L$ is the ground state decreases. ${ }^{8}$ Therefore, for a larger radius of the double ring configuration, we expect many more ground-state transitions. Figure 9 shows the ground-state free energy for a single inner ring with radii $R_{o}^{*}=2.0 \xi$ and $R_{i}^{*}=1.5 \xi$, for a single outer ring with radii $R_{o}=3.0 \xi$ and $R_{i}=2.6 \xi$ and for the coupled ring-ring configuration. The sample thickness is $d$ $=0.15 \xi$ and the Ginzburg-Landau parameter $\kappa=0.28$. For the single inner ring, the ground state changes from vorticity $L_{i n}=0$ up to $L_{i n}=10$ and the superconducting/normal transition field is at $H_{0} / H_{c 2}=6.73$. For the single outer ring the ground state changes from vorticity $L_{\text {out }}=0$ up to $L_{\text {out }}=32$ and superconductivity is destroyed at $H_{0} / H_{c 2}=8.40$. By comparing the free energy of the double ring with the one of the outer ring, we notice that there are many more groundstate transitions as a consequence of the transitions in the inner ring. For the single ring the minimum in the free energy of the $L+1$ state is always less negative than the one of the $L$ state. Due to the interaction between the two rings, this is no longer always the case for the double ring configuration. At $H_{0} / H_{c 2}>6.73$ the free energy of the double ring configuration equals the one of the outer ring since the inner ring is in the normal state.

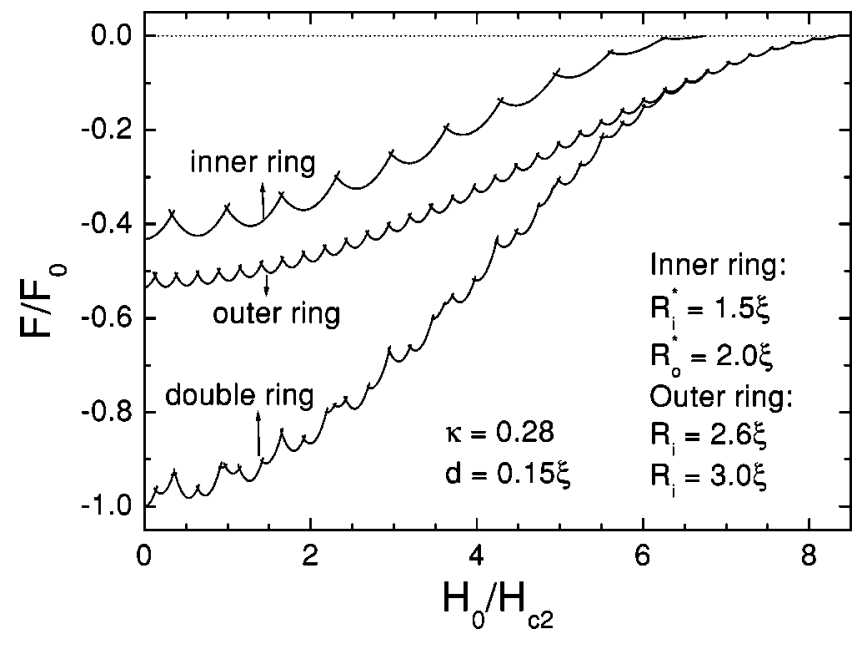

FIG. 9. The ground-state free energy for a single inner ring with radii $R_{o}^{*}=2.0 \xi$ and $R_{i}^{*}=1.5 \xi$, for a single outer ring with radii $R_{o}=3.0 \xi$ and $R_{i}=2.6 \xi$ and for the double ring configuration, i.e., the combination of these two rings. The sample thickness is $d$ $=0.15 \xi$ and the Ginzburg-Landau parameter $\kappa=0.28$.

Figures 10(a) and (b) show the field expelled from the region $\rho<R_{o}^{*}$ of the inner ring with radii $R_{o}^{*}=2.0 \xi$ and $R_{i}^{*}=1.5 \xi$ and the region $\rho<R_{o}$ of the outer ring with radii $R_{o}=3.0 \xi$ and $R_{i}=2.6 \xi$, respectively. The results for single rings are given by the dotted curves and for the double ring configuration by the solid curves. At low fields, the single inner ring is in the Meissner state and expels the magnetic field, i.e., $-M>0$. With increasing external field, more field

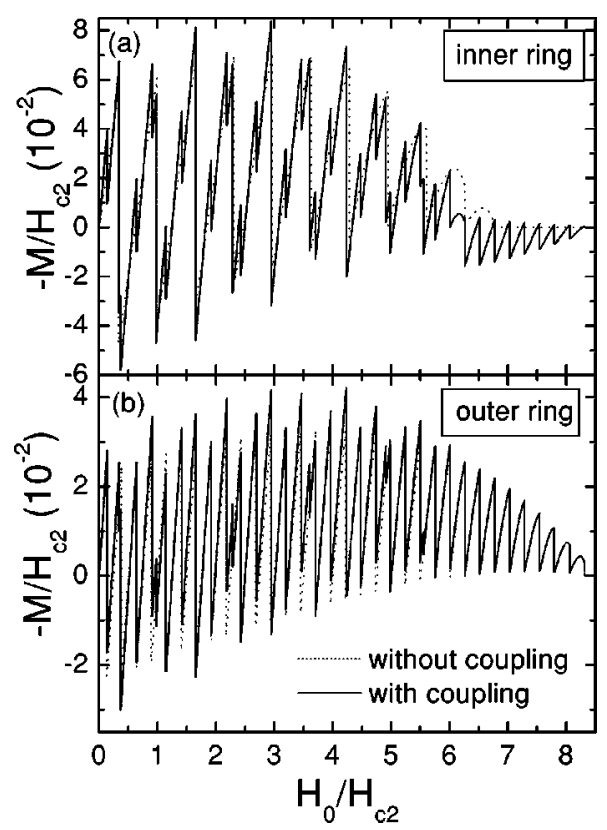

FIG. 10. The field expelled from the region $\rho<R_{o}^{*}$ of the inner ring with radii $R_{o}^{*}=2.0 \xi$ and $R_{i}^{*}=1.5 \xi(\mathrm{a})$, and the region $\rho<R_{o}$ of the outer ring with radii $R_{o}=3.0 \xi$ and $R_{i}=2.6 \xi$ (b). The results for single rings are given by the dotted curves and for the double ring configuration by the solid curves $(d=0.15 \xi$ and $\kappa=0.28)$. 


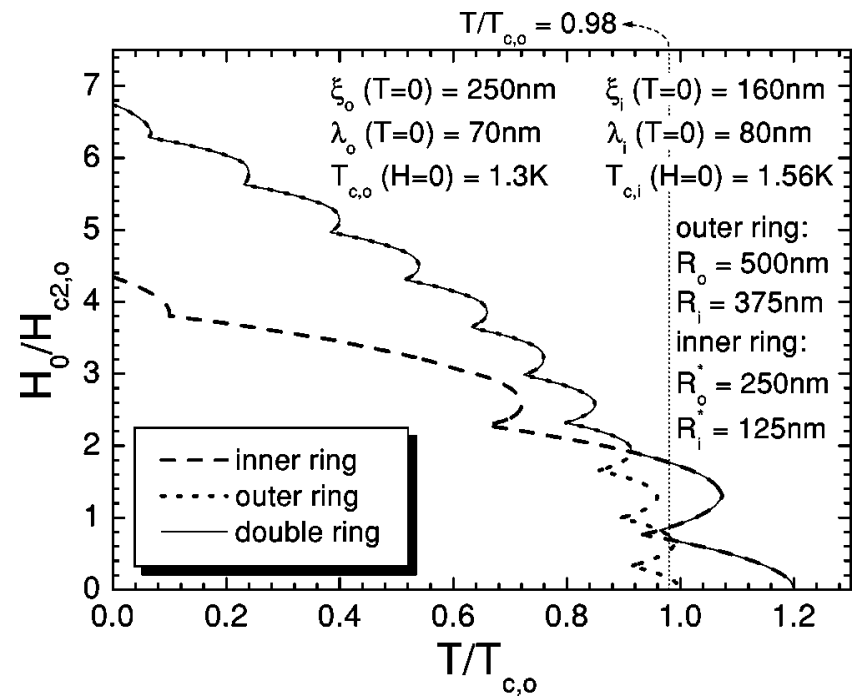

FIG. 11. The $H-T$ phase diagram for the inner ring (dashed curves), the outer ring (dotted curves), and the double ring configuration (solid curves). The material parameters and the sizes of both rings are different and are given in the figure.

is expelled. At $H_{0} / H_{c 2} \approx 0.33$ the ground state changes from $L_{i n}=0$ to $L_{i n}=1$ and flux has to be compressed into the hole to achieve vorticity 1 . Therefore the magnetic field inside the hole will be larger than the external one and $-M$ jumps to negative values, i.e., field compression. With increasing field, less flux has to be attracted and $-M$ becomes less negative. Further increasing the external flux becomes larger than the one needed for $L_{i n}=1$ and therefore the field has to be expelled again. This means that $-M$ becomes positive. Further increasing the field, more flux has to be expelled and $-M$ becomes more positive. At $H_{0} / H_{c 2} \approx 0.99$ the vorticity changes from $L_{i n}=1$ to $L_{i n}=2$, which means that $-M$ jumps to negative values, and so forth. At $H_{0} / H_{c 2}=6.73$ superconductivity is destroyed and the field becomes equal to the external one, and as a consequence, $-M=0$. The description for the single outer ring is completely analogous.

Placing a larger ring around the inner ring influences the expelled field drastically [see the solid curves in Fig. 10(a)]. Due to the expulsion of the outer ring at low fields, the magnetic field inside the hole of this ring will be smaller than the external one. Now, the expulsion by the inner ring results in a smaller local field than for the case of the single ring and, as a consequence, the expelled field increases at low fields. At $H_{0} / H_{c 2} \approx 0.13$ the ground state of the outer ring changes from vorticity $L_{\text {out }}=0$ to $L_{\text {out }}=1$, which means that suddenly the outer ring has to attract flux to achieve vorticity 1. Therefore the field inside the hole of the outer superconductor becomes larger than the external one and the expulsion by the inner ring will be less pronounced than for the case of the single ring. As a consequence, $-M$ jumps from a value above the one for the single ring case to a value below this value. This interplay between the two rings leads to a higher expulsion (attraction) from the region $\rho<R_{o}^{*}$ when the outer ring expels (attracts) flux and to a lower expulsion when the outer ring attracts (expels) flux. For the outer ring

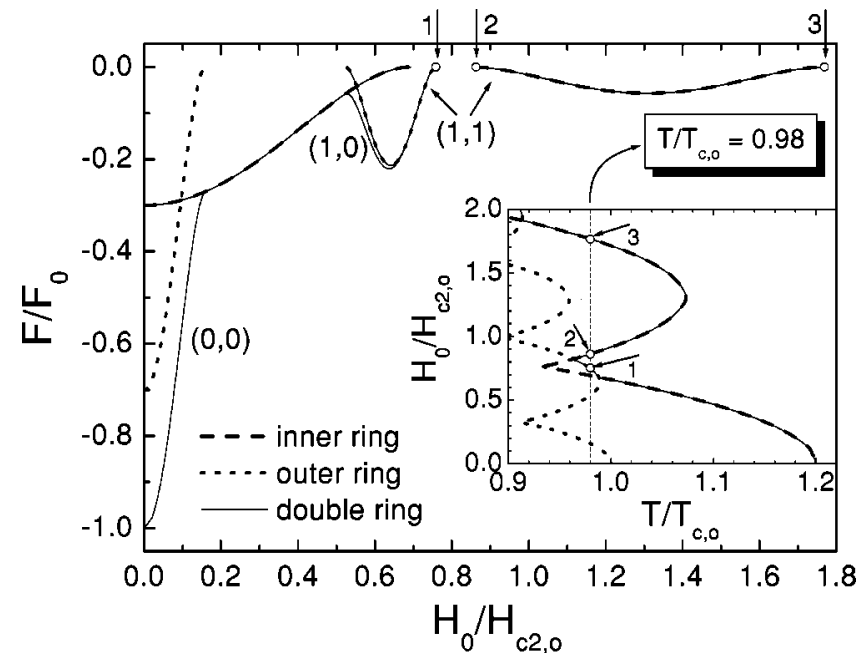

FIG. 12. The free energy as a function of the applied magnetic field for the inner ring (dashed curves), the outer ring (dotted curves), and the double ring configuration (solid curves) for the system of Fig. 11 at $T=0.98 T_{c, o}$. The inset shows an enlargement of the phase diagram (Fig. 11) in the $T / T_{c, o} \sim 1$ region.

an analogous explanation can be given [see the solid curves in Fig. 10(b)].

\section{TWO COUPLED RINGS OF DIFFERENT MATERIALS}

Until now, we considered always two superconductors made of the same material. This means that both superconductors have the same coherence length, penetration depth, and critical temperature, i.e., $\xi_{i}=\xi_{o}, \lambda_{i}=\lambda_{o}$, and $T_{c, i}$ $=T_{c, o}$. Since both rings have the same width and the radius of the inner ring is smaller than the one of the outer ring, the inner ring becomes normal at a smaller field than the outer ring. As a consequence, no effect of the magnetic coupling can be observed in the $H-T$ phase diagram. To circumvent this problem the $T_{c}$ of the outer ring was artificially lowered in the experiment of Ref. 29 by applying a sufficiently large external current through the outer ring. An alternative approach will be followed in the present section where we take the inner ring of a different material such that it has a higher critical temperature than the outer ring, and also a different coherence length and penetration depth, which leads to a different Ginzburg-Landau parameter.

As an example, we take for the outer ring the values used by Geim et al. ${ }^{14}$ for $\mathrm{Al}$, i.e., $\xi_{o}(T=0)=250 \mathrm{~nm}, \lambda_{o}(T$ $=0)=70 \mathrm{~nm}$, and thus $\kappa_{o}=0.28$, resulting in a critical temperature $T_{c, o}(H=0)=1.3 \mathrm{~K}$. For the inner ring, we assume a higher critical temperature $T_{c, i}=1.2 T_{c, o}=1.56 \mathrm{~K}$, and $\xi_{i}(T=0)=160 \mathrm{~nm}, \lambda_{i}(T=0)=80 \mathrm{~nm}$, and thus $\kappa_{i}=0.5$. For the radii of the outer ring we take as an example $R_{i}$ $=375 \mathrm{~nm}, R_{o}=500 \mathrm{~nm}$, and for the inner ring $R_{i}^{*}$ $=125 \mathrm{~nm}$ and $R_{o}^{*}=250 \mathrm{~nm}$. The $H-T$ phase diagram is shown in Fig. 11 for the uncoupled situation for the inner ring (thick dashed curves) and for the outer ring (thick dotted curves) and the coupled double ring situation (solid curve). At $T=0$ the outer ring has a much higher critical field 
$\left(H_{n u c} / H_{c 2, o}=6.74\right)$ than the inner ring $\left(H_{n u c} / H_{c 2, o}=4.34\right)$. Therefore the superconducting/normal transition of the double ring configuration equals the one of the outer ring for low temperatures. With increasing temperature, the nucleation field of the outer ring, i.e., the one of the double ring system, changes more quickly than the one of the inner ring. The oscillations are the well-known Little-Parks oscillations. At $T / T_{c, o}=0.912$ both single rings have the same transition field $H_{n u c} / H_{c 2 . o}=1.91$. At higher temperatures, the superconducting/normal transition is determined by the inner ring.

The situation where the critical field of the outer ring is larger than the one of the inner ring is exhaustively described in the previous sections. In Fig. 12 we show the free energy for the configuration of Fig. 11 at $T=0.98 T_{c, o}$ where the superconductivity of the inner ring exists at larger fields than the one of the outer ring. The free energy of the superconducting states of the inner ring are given by the dashed curves, the states of the outer ring by the dotted curves, and the double ring configuration by the solid curves. Both in the inner and the outer ring, superconducting states with vorticity $L=0$ and $L=1$ exist. At $T=0.98 T_{c, o}$, the critical fields of the inner and the outer ring are $H_{0} / H_{c 2, o}=1.77$ and 0.69 , respectively. Notice that in both rings the free energies of the $L=0$ state and the $L=1$ state do not cross, which means that with increasing field the ground state changes from the Meissner state into the normal state and, with further increasing the field, into the $L=1$ state and back into the normal state. The reason is that near $T_{c}$ the superconductivity of the ring has decreased. This means that only rather small currents can be induced and thus only a small flux can be attracted or expelled by the ring. In the region between the existence of the $L=0$ state and the $L=1$ state the currents, which have to be induced to expel or attract the necessary flux to achieve vorticity $L=0$ and $L=1$, are too high. With increasing temperature, the $L=1$ state cannot nucleate anymore and the superconducting/normal transition jumps to the field where the $L=0$ state is destroyed. The corresponding oscillations in the $H-T$ phase diagram (Fig. 11) are the LittleParks oscillations. For the double ring configuration the $(0,0)$, the $(1,0)$, and the $(1,1)$ state can nucleate. The $(1,1)$ state is split into two parts corresponding to the $L=1$ states in the two single rings with an intermediate magnetic-field region in which both superconductors are normal. The ground state changes from the Meissner state $(0,0)$ into the $(1,0)$ state at $H_{0} / H_{c 2, o} \approx 0.53$, which equals the $(1,1)$ state at $H_{0} / H_{c 2, o}>0.69$. Further increasing the field the ground state changes into the normal state at $H_{0} / H_{c 2, o} \approx 0.76$, then back into the $(1,1)$ state at $H_{0} / H_{c 2, o} \approx 0.86$ and further back into the normal state at $H_{0} / H_{c 2, o} \approx 1.77$. Compared to the uncoupled inner ring and the outer ring situation, extra groundstate transitions occur for the double ring case with interesting re-entrant superconducting behavior and a switching on and off of the superconducting state in the inner and outer ring.

\section{TWO COUPLED THIN RINGS IN THE LIMIT $\kappa \gg 1$}

Here we will show that in the limit of two coupled thin rings it is possible to obtain analytical results for the cou- pling energy between the two rings. This also corresponds to the case of $\kappa \gg 1$ and allows us to solve the problem analytically with the small parameter $d / \kappa^{2} \ll 1$. From the numerical calculations of previous sections it follows that the radial dependence of the order parameter in both inner and outer rings is slow and smooth. Therefore we assume that the order parameter in both inner and outer rings, $f_{\text {in }}$ and $f_{\text {out }}$, respectively, are constant. In the limit of the thin rings we neglect in the first approximation the $z$ dependence of the vector potential. With this assumption and because of the cylindrical symmetry of the problem the vector potential has only the azimuthal component $A(\rho)$ and the magnetic field has only the normal component $H(\rho)=\partial[\rho A(\rho)] / \partial \rho$.

The distribution of the vector potential due to the supercurrents inside the inner and outer ring are described by the following equation [see also, for example, Eqs. (2) and (3) in Ref. 18]:

$$
\begin{aligned}
& -\kappa_{i(o)}^{2} \frac{\partial}{\partial \rho}\left[\frac{1}{\rho} \frac{\partial}{\partial \rho}\left(\rho A_{\text {in }(\text { out })}\right)\right] \\
& =d\left(\frac{L_{\text {in }(\text { out })}}{\rho}-A_{\text {in }(\text { out })}\right) f_{\text {in }(\text { out })}^{2},
\end{aligned}
$$

and outside the rings by

$$
\frac{\partial}{\partial \rho}\left[\frac{1}{\rho} \frac{\partial}{\partial \rho}(\rho A)\right]=0
$$

The corresponding boundary conditions are the continuity of both $A(\rho)$ and $H(\rho)$ on the radial sides of the rings. For $d$ $\rightarrow 0$ it gives everywhere $A(d=0)=H_{0} \rho / 2$ and $H(d=0)$ $=H_{0}$. To a first approximation the solution of Eq. (13a) becomes, for small $d / \kappa_{i(o)}^{2}$,

$$
\begin{aligned}
A_{\text {in }(\text { out })}(\rho)= & \frac{H_{0} \rho}{2}+\frac{D_{\text {in }(\text { out })} \rho}{2}+\frac{C_{\text {in }(\text { out })}}{\rho} \\
& -\frac{d}{4 \kappa_{i(o)}^{2}} f_{\text {in }(\text { out })}^{2} \rho\left[L_{\text {in }(\text { out })}(2 \ln \rho-1)\right. \\
& \left.-H_{0} \rho^{2} / 4\right]
\end{aligned}
$$

which is valid inside the superconductors, i.e., in the range $R_{i}^{*}<\rho<R_{o}^{*}$ and $R_{i}<\rho<R_{o}$. The vector potential outside the rings is then obtained from a solution of Eq. (13b) and is equal to

$$
A(\rho)= \begin{cases}D_{1} \rho / 2, & 0 \leqslant \rho \leqslant R_{i}^{*}, \\ D_{3} \rho / 2+C_{3} / \rho, & R_{o}^{*} \leqslant \rho \leqslant R_{i}, \\ H_{0} \rho / 2+C_{e x t} / \rho, & \rho \geqslant R_{o} .\end{cases}
$$

The corresponding distribution of the magnetic field is 


$$
H(\rho)= \begin{cases}D_{1}, & 0 \leqslant \rho \leqslant R_{i}^{*}, \\ H_{0}+D_{\text {in }}-f_{\text {in }}^{2}\left(d / \kappa_{i}^{2}\right)\left(L_{\text {in }} \ln \rho-H_{0} \rho^{2} / 4\right), & R_{i}^{*} \leqslant \rho \leqslant R_{o}^{*}, \\ D_{3}, & R_{o}^{*} \leqslant \rho \leqslant R_{i}, \\ H_{0}+D_{\text {out }}-f_{\text {out }}^{2}\left(d / \kappa_{o}^{2}\right)\left[L_{\text {out }} \ln \rho-H_{0} \rho^{2} / 4\right], & R_{i} \leqslant \rho \leqslant R_{o}, \\ H_{0}, & \rho \geqslant R_{o} .\end{cases}
$$

From the above boundary conditions the integration constants $D$ and $C$ in Eqs. (14a)-(15) are

$$
\begin{gathered}
C_{\text {in }}=\left(d / 4 \kappa_{i}^{2}\right) f_{\text {in }}^{2} R_{i}^{* 2}\left(-L_{\text {in }}+H_{0} R_{i}^{* 2} / 4\right), \\
C_{3}=\left(d / 4 \kappa_{i}^{2}\right) f_{\text {in }}^{2}\left(R_{o}^{* 2}-R_{i}^{* 2}\right) \\
\times\left[L_{\text {in }}-H_{0}\left(R_{o}^{* 2}+R_{i}^{* 2}\right) / 4\right], \\
C_{\text {out }}=C_{3}+\left(d / 4 \kappa_{o}^{2}\right) f_{\text {out }}^{2} R_{i}^{2}\left(-L_{\text {out }}+H_{0} R_{i}^{2} / 4\right), \\
C_{\text {ext }}=C_{3}+\left(d / 4 \kappa_{o}^{2}\right) f_{\text {out }}^{2}\left(R_{o}^{2}-R_{i}^{2}\right) \\
\times\left[L_{\text {out }}-H_{0}\left(R_{o}^{2}+R_{i}^{2}\right) / 4\right], \\
D_{\text {out }}=\left(d / \kappa_{o}^{2}\right) f_{\text {out }}^{2}\left[L_{\text {out }} \ln R_{o}-H_{0} R_{o}^{2} / 4\right], \\
H_{0}+\left(d / \kappa_{o}^{2}\right) f_{\text {out }}^{2}\left[L_{\text {out }} \ln \left(R_{o} / R_{i}\right)-H_{0} S_{\text {out }} / 4 \pi\right], \\
D_{\text {in }}=\left(d / \kappa_{i}^{2}\right) f_{\text {in }}^{2}\left[L_{\text {in }} \ln R_{o}^{*}-H_{0} R_{o}^{* 2} / 4\right] \\
+\left(d / \kappa_{o}^{2}\right) f_{\text {out }}^{2}\left[L_{\text {out }} \ln \left(R_{o} / R_{i}\right)-H_{0} S_{\text {out }} / 4 \pi\right], \\
D_{1}=H_{0}+\left(d / \kappa_{i}^{2}\right) f_{\text {in }}^{2}\left[L_{\text {in }} \ln \left(R_{o}^{*} / R_{i}^{*}\right)\right. \\
\left.-H_{0} S_{\text {in }} / 4 \pi\right]+\left(d / \kappa_{o}^{2}\right) f_{\text {out }}^{2}\left[L_{\text {out }} \ln \left(R_{o} / R_{i}\right)\right. \\
\left.-H_{0} S_{\text {out }} / 4 \pi\right] .
\end{gathered}
$$

Notice that within this approximation the magnetic field is constant in the inner hole and in the space between the two rings, and has a small (as the ring's thickness is small) radial dependence inside the rings. Inserting the expressions for the order parameters, the magnetic field, and the vector potential into the expression for the energy [note that in the equivalent expression (7) the Ginzburg-Landau equations have already been used],

$$
\begin{aligned}
F= & \frac{2}{V} \int d V\left\{-\frac{\xi_{o}^{2}}{\xi_{i, o}^{2}}|\Psi|^{2}+\frac{1}{2} \frac{\kappa_{i, o}^{2}}{\kappa_{o}^{2}}|\Psi|^{4}\right. \\
& \left.+|-i \vec{\nabla} \Psi-\vec{A} \Psi|^{2}+\kappa_{o}^{2}\left[\vec{h}(\vec{r})-\vec{H}_{0}\right]^{2}\right\} .
\end{aligned}
$$

With a $O\left(d^{2} / \kappa^{4}\right)$ accuracy we find the difference between the Gibbs free energy of the superconducting state and the normal state,

$$
\begin{aligned}
F= & \frac{2}{S}\left[\left(-\frac{\xi_{o}^{2}}{\xi_{i}^{2}} f_{\text {in }}^{2}+\frac{1}{2} \frac{\kappa_{i}^{2}}{\kappa_{o}^{2}} f_{\text {in }}^{4}\right) S_{\text {in }}\right. \\
& +f_{\text {in }}^{2}\left(I_{\text {in }}^{(0)}-\left(d / \kappa_{i}^{2}\right) f_{\text {in }}^{2} I_{\text {in }}^{(1)}-\left(d / \kappa_{o}^{2}\right) f_{\text {out }}^{2} I^{(2)}\right) \\
& +\left(-f_{\text {out }}^{2}+\frac{1}{2} f_{\text {out }}^{4}\right) S_{\text {out }} \\
& \left.+f_{\text {out }}^{2}\left(I_{\text {out }}^{(0)}-\left(d / \kappa_{o}^{2}\right) f_{\text {out }}^{2} I_{\text {out }}^{(1)}-\left(d / \kappa_{i}^{2}\right) f_{\text {in }}^{2} I^{(2)}\right)\right],
\end{aligned}
$$

where $\quad S_{\text {in }}=\pi\left(R_{o}^{* 2}-R_{i}^{* 2}\right), \quad S_{\text {out }}=\pi\left(R_{o}^{2}-R_{i}^{2}\right), \quad S=S_{\text {in }}$ $+S_{\text {out }}$, and

$$
\begin{aligned}
I_{\text {out }(\text { in })}^{(0)}= & 2 \pi L_{\text {out }(\text { in })}^{2} \ln \left(R_{o}^{(*)} / R_{i}^{(*)}\right)-H_{0} L_{\text {out }(\text { in })} S_{\text {out }(\text { in })} \\
& +H_{0}^{2} S_{\text {out }(\text { in })}\left(R_{o}^{(*) 2}+R_{i}^{(*) 2}\right) / 8, \\
I^{(2)}= & S_{\text {in }}\left[L_{\text {in }}-H_{0}\left(R_{i}^{* 2}+R_{o}^{* 2}\right) / 4\right]\left[L_{\text {out }} \ln \left(R_{o} / R_{i}\right)\right. \\
& \left.-H_{0} S_{\text {out }} / 4 \pi\right], \\
I_{\text {out }(\text { in })}^{(1)}=2\left(L_{\text {out }(\text { in })} \ln R_{o}^{(*)}-H_{0} R_{o}^{(*) 2} / 4\right) J_{\text {out }(\text { in })}^{(1)} & \\
- & R_{i}^{(*) 2}\left(L_{\text {out }(\text { in })}-H_{0} R_{i}^{(*) 2} / 4\right) J_{\text {out }(\text { in })}^{(2)}-J_{\text {out }(\text { in })}^{(3)}, \\
J_{\text {out }(\text { in })}^{(1)}= & \frac{S_{\text {out }(\text { in })}}{2}\left[L_{\text {out }(\text { in })}-H_{0}\left(R_{o}^{(*) 2}+R_{i}^{(*) 2}\right) / 4\right], \\
J_{\text {out }(\text { in })}^{(2)}= & \pi\left[L_{\text {out }(\text { in })} \ln \left(R_{o}^{(*)} / R_{i}^{(*)}\right)-H_{0} S_{\text {out }(\text { in })} / 4 \pi\right], \\
J_{\text {out }(\text { in })}^{(3)}= & \pi \rho^{2}\left[L_{\text {out }(\text { in })}^{2}(\ln \rho-1)+L_{\text {out }(\text { in })} H_{0} \rho^{2}\right. \\
& \left.\times(1-2 \ln \rho) / 8+H_{0}^{2} \rho^{4} / 48\right]\left.\right|_{R_{i}^{(*)}} ^{R_{\text {o }}^{(*)}} .
\end{aligned}
$$

After the minimization of the free energy (17) with respect to $f_{\text {in(out })}$ we obtain two equations,

$$
\begin{gathered}
f_{\text {in }(\text { out })}\left[\left(-\frac{\xi_{o}^{2}}{\xi_{i(o)}^{2}}+\frac{\kappa_{i(o)}^{2}}{\kappa_{o}^{2}} f_{\text {in }(\text { out })}^{2}\right) S_{\text {in }(\text { out })}+I_{\text {in }(\text { out })}^{(0)}\right. \\
\left.-\frac{2 d}{\kappa_{i(o)}^{2}} f_{\text {in (out })}^{2} I_{\text {in }(\text { out })}^{(1)}-\left(\frac{d}{\kappa_{o}^{2}}+\frac{d}{\kappa_{i}^{2}}\right) f_{\text {out }(\text { in })}^{2} I^{(2)}\right]=0,
\end{gathered}
$$




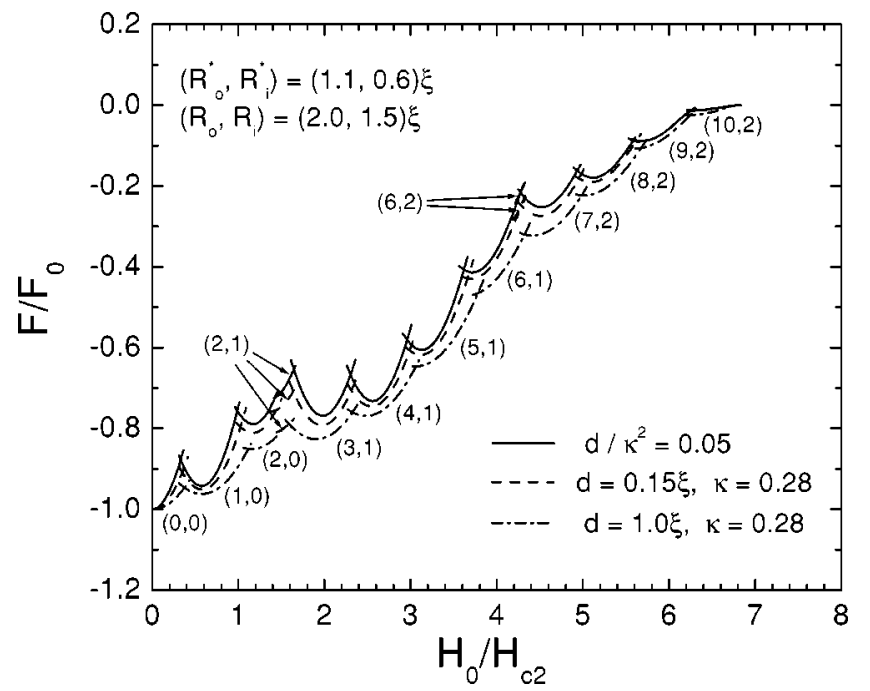

FIG. 13. The ground-state free energy of the double ring configuration with the same parameters as in Fig. 5 obtained from our analytical expressions which are valid for type-II superconducting rings. Also the ground-state energies from Fig. 5 are shown.

from which we obtain the equilibrium values of the order parameters for the three possible situations.

When both the inner and outer rings are in the superconducting state (case I), we obtain with a $O\left(d^{2} / \kappa^{4}\right)$ accuracy

$$
\begin{aligned}
\widetilde{f}_{\text {in }(\text { out })}^{2}= & \frac{\kappa_{o}^{2}}{\kappa_{i(o)}^{2}}\left(\frac{\xi_{o}^{2}}{\xi_{i(o)}^{2}}-\frac{I_{\text {in }(\text { out })}^{(0)}}{S_{\text {in }(\text { out })}}\right)\left(1+\frac{2 d}{\kappa_{i(o)}^{2}} \frac{\kappa_{o}^{2}}{\kappa_{i(o)}^{2}} \frac{I_{\text {in }(\text { out })}^{(1)}}{S_{\text {in }(\text { out })}}\right) \\
& +\frac{d}{\kappa_{i}^{2}}\left(1+\frac{\kappa_{o}^{2}}{\kappa_{i}^{2}}\right)\left(\frac{\xi_{o}^{2}}{\xi_{o(i)}^{2}}-\frac{I_{\text {out }(\text { in })}^{(0)}}{S_{\text {out }(\text { in })}}\right) \frac{I^{(2)}}{S_{\text {in }(\text { out })}}
\end{aligned}
$$

Inserting these expressions into Eq. (17) we obtain $F^{I}=F_{\text {in }}^{I}$ $+F_{\text {out }}^{I}+F_{\text {int }}^{I}$, where

$$
F_{i n}^{I}=-\frac{S_{i n}}{S} \frac{\kappa_{o}^{4}}{\kappa_{i}^{4}}\left(\frac{\xi_{o}^{2}}{\xi_{i}^{2}}-\frac{I_{i n}^{(0)}}{S_{i n}}\right)^{2}\left[\frac{\kappa_{i}^{2}}{\kappa_{o}^{2}}+\frac{2 d}{\kappa_{i}^{2}} \frac{I_{i n}^{(1)}}{S_{i n}}+O\left(\frac{d^{2}}{\kappa_{i}^{4}}\right)\right]
$$

is the self energy of the inner ring, and

$$
F_{\text {out }}^{I}=-\frac{S_{\text {out }}}{S}\left(1-\frac{I_{\text {out }}^{(0)}}{S_{\text {out }}}\right)^{2}\left[1+\frac{2 d}{\kappa_{o}^{2}} \frac{I_{\text {out }}^{(1)}}{S_{\text {out }}}+O\left(\frac{d^{2}}{\kappa_{o}^{4}}\right)\right],
$$

is the self energy of the outer ring, while

$$
\begin{aligned}
F_{\text {int }}^{I}= & -\frac{2 d}{\kappa_{i}^{2}}\left(1+\frac{\kappa_{o}^{2}}{\kappa_{i}^{2}}\right)\left(\frac{\xi_{o}^{2}}{\xi_{i}^{2}}-\frac{I_{\text {in }}^{(0)}}{S_{\text {in }}}\right) \\
& \times\left(1-\frac{I_{\text {out }}^{(0)}}{S_{\text {out }}}\right) \frac{I^{(2)}}{S}+O\left(\frac{d^{2}}{\kappa^{4}}\right),
\end{aligned}
$$

is the interaction energy between the two rings.
When one of the rings is in the normal state the results become more simple. Namely, when the outer ring is in the normal state and the inner ring is superconducting (case II) or vice versa (case III), we have

$$
\tilde{f}_{\text {out }}=0, \quad \widetilde{f}_{\text {in }}^{2}=\left(\frac{\xi_{o}^{2}}{\xi_{i}^{2}}-\frac{I_{\text {in }}^{(0)}}{S_{\text {in }}}\right)\left(\frac{\kappa_{i}^{2}}{\kappa_{o}^{2}}-\frac{2 d}{\kappa_{i}^{2}} \frac{I_{\text {in }}^{(1)}}{S_{\text {in }}}\right)^{-1},
$$

and

$$
\tilde{f}_{\text {in }}=0, \quad \widetilde{f}_{\text {out }}^{2}=\left(1-\frac{I_{\text {out }}^{(0)}}{S_{\text {out }}}\right)\left(1-\frac{2 d}{\kappa_{o}^{2}} \frac{I_{\text {out }}^{(1)}}{S_{\text {out }}}\right)^{-1}
$$

respectively. The corresponding energies are

$$
\begin{aligned}
& F^{I I}=-\frac{S_{\text {in }}}{S}\left(\frac{\xi_{o}^{2}}{\xi_{i}^{2}}-\frac{I_{\text {in }}^{(0)}}{S_{\text {in }}}\right)^{2}\left(\frac{\kappa_{i}^{2}}{\kappa_{o}^{2}}-\frac{2 d}{\kappa_{i}^{2}} \frac{I_{\text {in }}^{(1)}}{S_{\text {in }}}\right)^{-1}, \\
& F^{I I I}=-\frac{S_{\text {out }}}{S}\left(1-\frac{I_{\text {out }}^{(0)}}{S_{\text {out }}}\right)^{2}\left(1-\frac{2 d}{\kappa_{o}^{2}} \frac{I_{\text {out }}^{(1)}}{S_{\text {out }}}\right)^{-1},
\end{aligned}
$$

and in the $d / \kappa_{i(o)}^{2} \ll 1$ limit they coincide with $F_{i n}^{I}$ and $F_{o u t}^{I}$, respectively.

One can see that an interaction between the two rings (i.e., the coupling) exists only when both rings are superconducting. The energy of the ring-ring coupling in the considered limit is proportional to the ring's thickness. Due to the interaction between the rings the Cooper-pair density in each ring has a small (proportional to $d / \kappa^{2}$ ) contribution from the neighboring ring.

In Fig. 13 the calculated magnetic-field dependence of the ground-state energy of the coupled thin rings of the same material (i.e., with $\xi_{i}=\xi_{o}$ and $\kappa_{i}=\kappa_{o}$ ) with the same radial sizes as in Fig. 5 is shown for $d / \kappa^{2}=0.05$ (solid curve). Also for comparison the curves from Fig. 5 are shown by dashed and dash-dotted lines which correspond to $d / \kappa^{2} \simeq 1.98$ and 12.76, respectively. One can see that all curves have the same qualitative behavior and with increasing $d / \kappa^{2}$ only a small decrease of the ground-state energy takes place. Also the values of the transition fields between the different $L$ states are very nicely reproduced. This is quite surprising and, to make the physics of this result more clear, we consider the limit of narrow rings.

Let us introduce the average radius of the two rings, $\bar{\rho}_{\text {in }}$ $=\left(R_{o}^{*}+R_{i}^{*}\right) / 2, \bar{\rho}_{\text {out }}=\left(R_{o}+R_{i}\right) / 2$, respectively, and their corresponding widths, $2 w_{\text {in }}=R_{o}^{*}-R_{i}^{*}, 2 w_{\text {out }}=R_{o}-R_{i}$. Next we expand Eqs. (18) and (21) with respect to the small parameters $2 w_{\text {in (out) }} / \bar{\rho}_{\text {in (out })} \ll 1$ with an $O\left(w_{\text {in (out })}^{2}\right)$ accuracy. Within this approximation the energies (21) are 


$$
\begin{aligned}
F_{\text {in }(\text { out })}^{I}= & -\frac{S_{\text {in }(\text { out })}}{S} \frac{\kappa_{o(i)}^{2}}{\kappa_{i}^{2}}\left[\frac{\xi_{\text {o(i) }}^{2}}{\xi_{i}^{2}}-\frac{\left(L_{\text {in }(\text { out })}-\bar{\Phi}_{\text {in }(\text { out })}\right)^{2}}{\bar{\rho}_{\text {in }(\text { out })}^{2}}\right] \\
& \times\left[1+\frac{2 d}{\kappa_{i(o)}^{2}} \frac{\kappa_{o(i)}^{2}}{\kappa_{i}^{2}} \frac{2 w_{\text {in }(\text { out })}}{\bar{\rho}_{\text {in }(\text { out })}}\left(L_{\text {in }(\text { out })}-\bar{\Phi}_{\text {in }(\text { out })}\right)^{2}\right. \\
& \left.+O\left(\frac{d}{\kappa_{\text {i(o })}^{2}} w_{\text {in }(\text { out })}^{2}\right)\right]\left[\frac{\xi_{o(i)}^{2}}{\xi_{i}^{2}}\right. \\
& \left.-\frac{\left(L_{\text {in }(\text { out })}-\bar{\Phi}_{\text {in }(\text { out })}\right)^{2}}{\bar{\rho}_{\text {in }(\text { out })}^{2}}+O\left(w_{\text {in }(\text { out })}^{2}\right)\right], \\
F_{\text {int }}^{I}=- & \frac{S_{\text {in }}}{S} \frac{2 d}{\kappa_{i}^{2}}\left\{\frac{2 w_{\text {out }}}{\bar{\rho}_{\text {out }}}\left(1+\frac{\kappa_{o}^{2}}{\kappa_{i}^{2}}\right)\left(L_{\text {in }}-\bar{\Phi}_{\text {in }}\right)\left(L_{\text {out }}-\bar{\Phi}_{\text {out }}\right)\right. \\
\times & {\left[\frac{\xi_{o}^{2}}{\xi_{i}^{2}}-\frac{\left(L_{\text {in }}-\bar{\Phi}_{\text {in }}\right)^{2}}{\bar{\rho}_{\text {in }}^{2}}\right]\left[1-\frac{\left(L_{\text {out }}-\bar{\Phi}_{\text {out }}\right)^{2}}{\bar{\rho}_{\text {out }}^{2}}\right] } \\
+ & \left.O\left(w_{\text {in }} w_{\text {out }}, w_{\text {out }}^{2}\right)\right\},
\end{aligned}
$$

where $\bar{\Phi}_{\text {in(out) }}=H_{0} \bar{\rho}_{\text {in (out })}^{2} / 2$ is the average magnetic flux through the corresponding ring, measured in units of $\Phi_{0}$ $=h c / 2 e$. From these expressions one can see that the dominant thickness dependent terms in $F_{\text {in(out })}^{I}$, as well as the ring's interaction energy $F_{\text {int }}^{I}$, are of order $O\left[\left(d / \kappa_{i(o)}^{2}\right) w_{\text {in(out })}\right]$ [not of order $O\left(d / \kappa_{i(o)}^{2}\right)$, as one could think naively]. This additional smallness shows that an increase of the ring thickness influences the ring's self-energies and the interaction energy very slightly and for rings which have a not too large width (as in our case) the above results become valid for $d / \kappa_{i(o)}^{2} \geq 1$.

\section{CONCLUSIONS}

We investigated the magnetic coupling between two concentric mesoscopic superconductors with nonzero thickness. When a second superconductor is placed in the center of a superconducting ring, it feels a nonuniform field, which is the superposition of the uniform applied field and the field expelled from the outer ring. Also the first ring will be influenced by the magnetic field expelled from the superconductor in the center. So, both superconductors are coupled magnetically. This results in substantial changes of the superconducting properties.

From the study of the free energy we learned that extra ground-state transitions occur in comparison with the single ring case. These are transitions where the total vorticity stays the same, but the vorticity of the inner superconductor changes by one unit. We also found that the free energy of the double ring system is not exactly the same as the sum of the free energies of the two uncoupled single rings which is another signature of the magnetic coupling of both rings. This interaction enhances with increasing sample thickness. We also calculated the expelled field for the ring-ring configuration which showed that as compared with a single ring more, or less, field can be expelled or attracted depending on the vorticities of both superconductors.

The behavior of the Cooper-pair density, the magneticfield profile, and the current density was calculated. Since an extra superconductor is placed in the center, the magnetic field will be expelled from this superconductor or will be compressed in the center of it, which results in a higher or a lower magnetic-field density between the two superconductors. The current in both rings exhibits extra jumps at the transition fields where the vorticity of the other ring increases or decreases by 1 . The reason is that at these applied fields the total magnetic field in the region between the two superconductors changes.

We investigated what happens if the inner ring is made of a different material with a higher critical temperature. The $H-T$ phase diagram showed that the nucleation field of the double ring equals the one of the outer ring at low temperatures and the one of the inner ring at higher temperatures.

Analytical expressions are obtained for the magnetic-field distribution and the energy of two coupled thin type-II superconducting rings. These analytical results are found to give excellent results when $d / \kappa_{i(o)}^{2}<1$ and, moreover, give also good agreement with our "exact" numerical results for sufficiently narrow rings when $d / \kappa_{i(o)}^{2}>1$.

\section{ACKNOWLEDGMENTS}

This work was supported by the Flemish Science Foundation (FWO-Vl), the "Onderzoeksraad van de Universiteit Antwerpen" (GOA), the "Interuniversity Poles of Attraction Program-Belgian State, Prime Minister's Office-Federal Office for Scientific, Technical and Cultural Affairs," and the ESF "VORTEX" program. Discussions with V. V. Moshchalkov and M. Morelle are gratefully acknowledged.
*Permanent address: Donetsk Physical \& Technical Institute, National Academy of Sciences of Ukraine, Donetsk 83114, Ukraine.

†Electronic mail: francois.peeters@ua.ac.be

${ }^{1}$ For a review, see, for example, Jorge Berger and Jacob Rubinstein, Connectivity and Superconductivity (Springer, Berlin, 2000).

${ }^{2}$ H. Vloeberghs, V.V. Moshchalkov, C. Van Haesendonck, R. Jonckheere, and Y. Bruynseraede, Phys. Rev. Lett. 69, 1268 (1992).

${ }^{3}$ J. Berger and J. Rubinstein, Phys. Rev. Lett. 75, 320 (1995); Phys. Rev. B 56, 5124 (1997); 59, 8896 (1999).

${ }^{4}$ V.V. Moshchalkov, L. Gielen, C. Strunk, R. Jonckheere, X. Qiu,
C. van Haesendonck, and Y. Bruynseraede, Nature (London) 373, 319 (1995).

${ }^{5}$ E.M. Horane, J.J. Castro, G.C. Buscaglia, and A. Lopez, Phys. Rev. B 53, 9296 (1996).

${ }^{6}$ V.M. Fomin, V.R. Misko, J.T. Devreese, and V.V. Moshchalkov, Solid State Commun. 101, 303 (1997); Phys. Rev. B 58, 11703 (1998).

${ }^{7}$ V. Bruyndoncx, L. Van Look, M. Verschuere, and V.V. Moshchalkov, Phys. Rev. B 60, 10468 (1999); V. Bruyndoncx, L. Van Look, and V.V. Moshchalkov, Physica C 332, 417 (2000).

${ }^{8}$ B.J. Baelus, F.M. Peeters, and V.A. Schweigert, Phys. Rev. B 61, 
9734 (2000); F.M. Peeters, V.A. Schweigert, B.J. Baelus, and P.S. Deo, Physica C 332, 255 (2000).

${ }^{9}$ B.J. Baelus, F.M. Peeters, and V.A. Schweigert, Phys. Rev. B 63, 144517 (2001).

${ }^{10}$ S.V. Yampolskii, F.M. Peeters, B.J. Baelus, and H.J. Fink, Phys. Rev. B 64, 052504 (2001).

${ }^{11}$ S. Pedersen, G.R. Kofod, J.C. Hollingbery, C.B. Sørensen, and P.E. Lindelof, Phys. Rev. B 64, 104522 (2001).

${ }^{12}$ O. Buisson, P. Gandit, R. Rammel, Y.Y. Wang, and B. Pannetier, Phys. Lett. A 150, 36 (1990).

${ }^{13}$ R. Benoist and W. Zwerger, Z. Phys. B: Condens. Matter 103, 377 (1997).

${ }^{14}$ A.K. Geim, I.V. Grigorieva, S.V. Dubonos, J.G.S. Lok, J.C. Maan, A.E. Filippov, and F.M. Peeters, Nature (London) 390, 256 (1997).

${ }^{15}$ P.S. Deo, V.A. Schweigert, F.M. Peeters, and A.K. Geim, Phys. Rev. Lett. 79, 4653 (1997); P.S. Deo, F.M. Peeters, and V.A. Schweigert, Superlattices Microstruct. 25, 1195 (1999).

${ }^{16}$ A.K. Geim, S.V. Dubonos, J.G.S. Lok, M. Henini, and J.C. Maan, Nature (London) 396, 144 (1998).

${ }^{17}$ V.A. Schweigert and F.M. Peeters, Phys. Rev. B 57, 13817 (1998).
${ }^{18}$ V.A. Schweigert, F.M. Peeters, and P.S. Deo, Phys. Rev. Lett. 81, 2783 (1998).

${ }^{19}$ V.A. Schweigert and F.M. Peeters, Physica C 332, 426 (2000).

${ }^{20}$ J.J. Palacios, Physica B 256-258, 610 (1998); Phys. Rev. B 58, R5948 (1998); Phys. Rev. Lett. 84, 1796 (2000).

${ }^{21}$ V.A. Schweigert and F.M. Peeters, Phys. Rev. Lett. 83, 2409 (1999).

${ }^{22}$ S.V. Yampolskii and F.M. Peeters, Phys. Rev. B 62, 9663 (2000).

${ }^{23}$ E. Akkermans, D.M. Gangardt, and K. Mallick, Phys. Rev. B 62, 12427 (2000); E. Akkermans and K. Mallick, Physica C 332, 250 (2000).

${ }^{24}$ A.K. Geim, S.V. Dubonos, J.J. Palacios, I.V. Grigorieva, M. Henini, and J.J. Schermer, Phys. Rev. Lett. 85, 1528 (2000).

${ }^{25}$ S.V. Yampolskii, B.J. Baelus, F.M. Peeters, and J. Koláček, Phys. Rev. B 64, 144511 (2001).

${ }^{26}$ J. Wang and Z.S. Ma, Phys. Rev. B 52, 14829 (1995).

${ }^{27}$ L.P. Levy, G. Dolan, J. Dunsmuir, and H. Bouchiat, Phys. Rev. Lett. 64, 2074 (1990).

${ }^{28}$ D. Davidović, S. Kumar, D.H. Reich, J. Siegel, S.B. Field, R.C. Tiberio, R. Hey, and K. Ploog, Phys. Rev. Lett. 76, 815 (1996); Phys. Rev. B 55, 6518 (1997).

${ }^{29}$ M. Morelle, V. Bruyndoncx, R. Jonckheere, and V.V. Moshchalkov, Phys. Rev. B 64, 064516 (2001). 